\title{
Hecke group algebras as degenerate affine Hecke algebras
}

\author{
Florent Hivert $^{1}$ and Anne Schilling ${ }^{2}$ and and Nicolas M. Thiéry ${ }^{2,3}$ \\ ${ }^{1}$ LITIS (EA 4108), Université de Rouen, Avenue de l'Université BP12 76801 Saint-Etienne du Rouvray, France and \\ Institut Gaspard Monge (UMR 8049) ${ }^{2}$ Department of Mathematics, University of California, One Shields Avenue, \\ Davis, CA 95616, U.S.A. \\ ${ }^{3}$ Univ Paris-Sud, Laboratoire de Mathématiques d'Orsay, Orsay, F-91405; CNRS, Orsay, F-91405, France
}

\begin{abstract}
The Hecke group algebra $\mathrm{H} \stackrel{\circ}{W}$ of a finite Coxeter group $\stackrel{\circ}{W}$, as introduced by the first and last author, is obtained from $\mathscr{D}^{\circ}$ by gluing appropriately its 0 -Hecke algebra and its group algebra. In this paper, we give an equivalent alternative construction in the case when ${ }^{\circ}$ is the classical Weyl group associated to an affine Weyl group $W$. Namely, we prove that, for $q$ not a root of unity, $\mathrm{H} \stackrel{\circ}{W}$ is the natural quotient of the affine Hecke algebra $\mathrm{H}(W)(q)$ through its level 0 representation. The proof relies on the following core combinatorial result: at level 0 the 0 -Hecke algebra acts transitively on $\stackrel{W}{W}$. Equivalently, in type $A$, a word written on a circle can be both sorted and antisorted by elementary bubble sort operators. We further show that the level 0 representation is a calibrated principal series representation $M(t)$ for a suitable choice of character $t$, so that the quotient factors (non trivially) through the principal central specialization. This explains in particular the similarities between the representation theory of the classical 0-Hecke algebra and that of the affine Hecke algebra at this specialization.

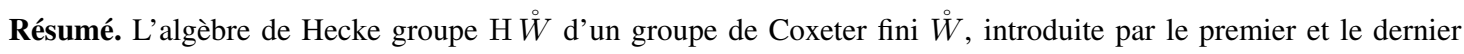
auteur, est obtenue en recollant de manière appropriée son algèbre de Hecke dégénérée et son algèbre de groupe. Dans cet article, nous donnons une construction alternative dans le cas où $\stackrel{\circ}{W}$ est un groupe de Weyl associé à un groupe de Weyl affine $W$. Plus précisément, nous montrons que quand $q$ n'est ni nul ni une racine de l'unité, $\mathrm{H} \stackrel{\circ}{W}^{\text {est }}$ le quotient naturel de l'algèbre de Hecke affine $\mathrm{H}(W)(q)$ dans sa représentation de niveau 0 . Nous montrons de plus que la représentation de niveau 0 est une représentation de série principale calibrée $M(t)$ pour un certain caractère $t$, de sorte que le quotient se factorise par la spécialisation centrale principale. Ce fait explique en particulier les similarités entre les théories des représentations de l'algèbre de Hecke dégénérée et de l'algèbre de Hecke affine sous cette spécialisation.
\end{abstract}

Keywords: Coxeter groups, (affine) Weyl groups, (affine) Hecke algebras

\section{Introduction}

The starting point of this research lies in the striking similarities between the representation theories of the degenerate (Iwahori-)Hecke algebras on one side and of the principal central specialization of the affine Hecke algebras on the other. For the sake of simplicity, we describe those similarities for type A in this 
introduction, but they carry over straightforwardly to any affine Weyl group $W$ and its associated classical Weyl group $\stackrel{\circ}{W}$.

The representation theory of the degenerate Hecke algebras $\mathrm{H}_{n}(0)$ for general type has been worked out by Norton [Nor79] and special combinatorial features of type A have been described by Carter [Car86]. In particular, the projective modules $P_{I}$ of the type $A$ degenerate Hecke algebra $\mathrm{H}_{n}(0)$ are indexed by subsets $I$ of $\{1, \ldots, n-1\}$, and the basis of each $P_{I}$ is indexed by those permutations of $n$ whose descent set is $I$.

On the other hand, the classification of the irreducible finite dimensional representations of the affine Hecke algebra is due to Zelevinsky [Zel80]. They are indexed by simple combinatorial objects called multisegments. However, in this work, we are interested in a particular subcategory related to a central specialization for which the multisegments are also in bijection with subsets of $\{1, \ldots, n-1\}$. This relation is as follows. It is well known from Bernstein-Zelevinsky [BZ77] and Lusztig [Lus83], that the center of the affine Hecke algebra is the ring of symmetric polynomials $\mathbb{C}\left[Y_{1}, \ldots, Y_{n}\right]^{\mathfrak{S}_{n}}$ in some particular elements $Y_{1}, \ldots, Y_{n}$ such that as vector space,

$$
\widetilde{\mathrm{H}}_{n}(q) \simeq \mathrm{H}_{n}(q) \otimes \mathbb{C}\left[Y_{1}, \ldots, Y_{n}\right] .
$$

As a center, it acts by scalar multiplication in all irreducible representations, and one way to select a particular class of representations is to specialize the center in the algebra itself. Thus any ring morphism from $\mathbb{C}\left[Y_{1}, \ldots, Y_{n}\right]^{\mathfrak{S}_{n}}$ to $\mathbb{C}$, or in other words any scalar alphabet, defines a quotient of the affine Hecke algebra of dimension

$$
\operatorname{dim}\left(\mathrm{H}_{n}(q)\right) \operatorname{dim}\left(\mathbb{C}\left[Y_{1}, \ldots, Y_{n}\right] / \mathbb{C}\left[Y_{1}, \ldots, Y_{n}\right]^{\mathfrak{S}_{n}}\right)=n !^{2} .
$$

Let us denote by $\mathcal{H}_{n}(q)$ the quotient of $\widetilde{\mathrm{H}}_{n}(q)$ obtained by the principal specialization of its center to the alphabet $\frac{1-q^{n}}{1-q}:=\left\{1, q, \ldots, q^{n-1}\right\}$, that is

$$
\mathcal{H}_{n}(q):=\widetilde{\mathrm{H}}_{n}(q) /\left\langle e_{i}\left(Y_{1}, \ldots, Y_{n}\right)-e_{i}\left(1, q, \ldots, q^{n-1}\right) \mid i=1, \ldots, n\right\rangle,
$$

where $e_{i}$ denote the elementary symmetric polynomials. Then, in this particular case, the multisegments of Zelevinsky are in bijection with subsets $I$ of $\{1, \ldots, n-1\}$ and the irreducible representations $S_{I}$ of $\mathcal{H}_{n}(q)$ have their bases indexed by descent classes of permutations. Thus one expects a strong link between $\mathrm{H}_{n}(0)$ and $\mathcal{H}_{n}(q)$.

The goal of this paper is to explain this relation by means of the Hecke group algebra $\mathrm{H} W$ introduced by the first and the last authors [HT06, HT07]. Indeed, by definition, HW contains naturally the degenerated Hecke algebra $\mathrm{H}(\stackrel{\circ}{W})(0)$ and it was shown that the simple modules of $\mathrm{H} \stackrel{\circ}{W}$, when restricted to $\mathrm{H}(\stackrel{\circ}{W})(0)$ form a complete family of projective ones. The relation comes from the fact that there is a natural surjective morphism from the affine Hecke algebra $\mathrm{H}(W)(q)$ to $\mathrm{H} \stackrel{\circ}{W}$. As a consequence the simple modules of $\mathrm{H} \stackrel{\circ}{\circ}$ are also simple modules of $\mathrm{H}(W)(q)$ elucidating the similarities. This can be restated as follows:

Theorem 1.1 For q not a root of unity, there is a particular finite dimensional quotient $\mathrm{H} W$ of the affine Hecke algebra $\mathrm{H}(W)(q)$ which contains the 0 -Hecke algebra $\mathrm{H}(\stackrel{\circ}{W})(0)$ and such that any simple $\mathrm{H} W$ module is projective when restricted to $\mathrm{H}(\stackrel{\circ}{W})(0)$.

The remainder of this paper is structured as follows. 
In Sections 2 and 3 , we briefly review the required material on Coxeter groups, Hecke algebras, and Hecke group algebras, as well as on the central theme of this article: the level 0 action of an affine Weyl group $W$ on the associated classical Weyl group $W^{\circ}$ and the corresponding level 0 representation of the affine Hecke algebra on $\mathbb{C} W$.

In Section 4 , we prove the core combinatorial property (Theorem 4.2 which states that an affine 0Hecke algebra acts at level zero transitively on the classical chambers (or equivalently on the classical Weyl group). We first treat type $A$ where Theorem 4.2 states that a word written on a circle can be both sorted and antisorted by elementary bubble sort operators (explicit (anti)sorting algorithms are also provided for types B,C, and D). We proceed with a type-free geometric proof of Theorem 4.2 The ideas used in the proof are inspired by private notes on finite-dimensional representations of quantized affine algebras by Kashiwara [Kas08], albeit reexpressed in terms of alcove paths. In Section 5, we derive new sets of generators for the Hecke group algebra of a finite Weyl group, and in Section 6 we prove the main result of the paper, namely that for generic $q$, the Hecke group algebra is the natural quotient of the (extended) affine Hecke algebra through its representation at level 0 (Theorem 6.1). Interestingly enough, and unlike for the affine Weyl group $W$, the torus $Y$ does not degenerate trivially. In Section 7 , we describe exactly this degeneracy, and show that, for a suitable choice of character on $Y$, the level 0 representation is a calibrated principal series representation (Theorem 7.1). This allows to refine Theorem 6.1 to $q$ not a root of unity. Altogether, Theorems 6.1 and 7.1 can be interpreted as two new equivalent alternative constructions of the Hecke group algebra, while the latter provides a parametrized family of maximal decompositions of its identity into idempotents (Corollary 7.3).

A long version of this extended abstract, containing proofs, further figures, and applications, is available at arXiv:0804.3781v1 [math.RT].

\section{Coxeter groups, Hecke algebras, and Hecke group algebras}

In this and the next section, we briefly recall the notations and properties of Coxeter groups, Weyl groups, their Hecke and Hecke group algebras that we need in the sequel. For further reading on those topics, we refer the reader to [Hum90, Kac90, BB05].

\subsection{Coxeter groups and their geometric representations}

Let $W$ be a Coxeter group and $I$ the index set of its Dynkin diagram. Denote by $s_{i}$ for $i \in I$ its simple reflections and $w_{0}$ its maximal element (when $W$ finite). For $J \subset I$, write $W_{J}$ for the parabolic subgroup generated by $\left(s_{i}\right)_{i \in J}$. The left and right descent sets of an element $w \in W$ are respectively $\mathrm{D}_{L}(w)$ and $\mathrm{D}_{R}(w)$.

Fixing a (generalized) Cartan matrix $M=\left(m_{i, j}\right)_{i, j \in I}$ for $W$ with coefficients in a ring $\mathbb{K} \subset \mathbb{R}$, allows for realizing $W$ geometrically. Take the module $\mathfrak{h}^{*}:=\mathfrak{h}_{\mathbb{K}}^{*}:=\bigoplus_{i \in I} \mathbb{K} \alpha_{i}$ and its $\mathbb{K}$-dual $\mathfrak{h}:=$ $\mathfrak{h}_{\mathbb{K}}:=\bigoplus_{i \in I} \mathbb{K} \Lambda_{i}^{\vee}$, with the natural pairing $\left\langle\Lambda_{i}^{\vee}, \alpha_{j}\right\rangle=\delta_{i j}$. The $\alpha_{i}$ are the simple roots, and the $\Lambda_{i}^{\vee}$ the fundamental coweights. The simple coroots are given by $\alpha_{i}^{\vee}:=\sum_{j} m_{i, j} \Lambda_{i}^{\vee}$. The Coxeter group acts on $\mathfrak{h}$ by the number game:

$$
s_{i}\left(x^{\vee}\right):=x^{\vee}-\left\langle x^{\vee}, \alpha_{i}\right\rangle \alpha_{i}^{\vee} \text { for } x^{\vee} \in \mathfrak{h},
$$

and on $\mathfrak{h}^{*}$ by the dual number game:

$$
s_{i}(x):=x-\left\langle\alpha_{i}^{\vee}, x\right\rangle \alpha_{i} \quad \text { for } x \in \mathfrak{h}^{*} .
$$


Denote by $R:=\left\{w\left(\alpha_{i}\right) \mid w \in W, i \in I\right\}$ the set of roots, and by $R^{\vee}:=\left\{w\left(\alpha_{i}^{\vee}\right) \mid w \in W, i \in I\right\}$ the set of coroots. To each root $\alpha$ corresponds the reflection $s_{\alpha}$ across the associated coroot $\alpha^{\vee}$ and along the hyperplane $H_{\alpha}$ which splits $\mathfrak{h}$ into a positive $H_{\alpha}^{+}$and a negative half-space $H_{\alpha}^{-}$:

$$
H_{\alpha}:=\left\{x^{\vee} \in \mathfrak{h} \mid\left\langle x^{\vee}, \alpha\right\rangle=0\right\} \quad H_{\alpha}^{+}:=\left\{x^{\vee} \in \mathfrak{h} \mid\left\langle x^{\vee}, \alpha\right\rangle>0\right\} \quad H_{\alpha}^{-}:=\left\{x^{\vee} \in \mathfrak{h} \mid\left\langle x^{\vee}, \alpha\right\rangle<0\right\} .
$$

Take now $\mathbb{K}=\mathbb{R}$. Define the fundamental chamber as the open simplicial cone $C:=\left\{x^{\vee}\left|\left\langle x^{\vee}, \alpha_{i}\right\rangle\right\rangle\right.$ $0, \forall i \in I\}$. For each root $\alpha$, the fundamental chamber $C$ lies either entirely in $H_{\alpha}^{+}$or in $H_{\alpha}^{-} ; R$ splits accordingly into the sets of positive roots $R^{+}$and of negative roots $R^{-}$, and similarly for coroots.

The closure $\bar{C}$ of $C$ is a fundamental domain for the action of $W$ on the Tits cone $U:=\bigcup_{w \in W} w(\bar{C})$, and the elements $w$ of $W$ are in bijection with the chambers $w(C)$. This bijection induces both a left and a right action of $W$ on the chambers. The right action is particularly nice as the chambers $w(C)$ and $w(C) \cdot s_{i}=w s_{i}(C)$ share a common wall. Any sequence $i_{1}, \ldots, i_{r}$ gives therefore rise to a sequence of adjacent chambers $C, s_{i_{1}}(C), s_{i_{1}} s_{i_{2}}(C), \ldots, s_{i_{1}} \cdots s_{i_{r}}(C)$ from $C$ to $w(C)$ (where $w=s_{i_{1}} \cdots s_{i_{r}}$ ), called a gallery. By abuse of notation we denote the gallery also by $i_{1}, \ldots, i_{r}$.

\section{2 (Iwahori)-Hecke algebras}

Let $W$ be a Coxeter group and $q_{1}$ and $q_{2}$ two complex numbers. When defined, set $q=:-\frac{q_{1}}{q_{2}}$. The (generic, Iwahori) $\left(q_{1}, q_{2}\right)$-Hecke algebra $\mathrm{H}(W)\left(q_{1}, q_{2}\right)$ of $W$ is the $\mathbb{C}$-algebra generated by the operators $T_{i}$ subject to the same braid-like relations as the operators $s_{i}$ of $W$, and to the quadratic relation $\left(T_{i}-\right.$ $\left.q_{1}\right)\left(T_{i}-q_{2}\right)=0$. Its dimension is $|W|$, and a basis is given by the elements $T_{w}:=T_{i_{1}} \cdots T_{i_{r}}$ where $w \in W$ and $i_{1}, \ldots, i_{r}$ is a reduced word for $w$.

At $q_{1}=1, q_{2}=-1$ (so $q=1$ ), we recover the usual group algebra $\mathbb{C}[W]$ of $W$; in general, when $q_{1}+q_{2}=0$ one still recovers $\mathbb{C}[W]$ up to a scaling of the generators: $s_{i}=\frac{1}{q_{1}} T_{i}$. On the opposite side, taking $q_{1}=0$ and $q_{2} \neq 0$ (so $q=0$ ) yields the 0-Hecke algebra; it is also a monoid algebra for the 0 -Hecke monoid $\left\{\pi_{w} \mid w \in W\right\}$ generated by the idempotents $\pi_{i}:=\frac{1}{q_{2}} T_{i}$. At $q_{1}=q_{2}=0$, one obtains the NilCoxeter algebra. Traditionally, and depending on the application in mind, different authors choose different specializations of $q_{1}$ and $q_{2}$, typically $q_{1}=q$ and $q_{2}=-1$ (cf. [Wik08]), or $q_{1}=t^{\frac{1}{2}}$ and $q_{2}=t^{-\frac{1}{2}}$ (cf. for example [RY08]). For our needs, keeping the two eigenvalues generic yields more symmetrical formulas which are also easier to specialize to other conventions.

Define the unique operators $\bar{T}_{i}$ such that $T_{i}+\bar{T}_{i}=q_{1}+q_{2}$. They satisfy the same relations as the $T_{i}$, and further $T_{i} \bar{T}_{i}=\bar{T}_{i} T_{i}=q_{1} q_{2}$.

We may realize the 0-Hecke monoid geometrically on $\mathfrak{h}$ as follows. For each $i \in I$, define the (halflinear) idempotent $\pi_{i}$ (resp. $\bar{\pi}_{i}$ ) which projects onto the negative (resp. positive) half space with respect to the root $\alpha_{i}$ :

$$
\pi_{i}\left(x^{\vee}\right):=\left\{\begin{array}{ll}
s_{i}\left(x^{\vee}\right) & \text { if }\left\langle x^{\vee}, \alpha_{i}\right\rangle>0, \\
x^{\vee} & \text { otherwise; }
\end{array} \bar{\pi}_{i}\left(x^{\vee}\right):= \begin{cases}s_{i}\left(x^{\vee}\right) & \text { if }\left\langle x^{\vee}, \alpha_{i}\right\rangle<0, \\
x^{\vee} & \text { otherwise. }\end{cases}\right.
$$

As the reflection $s_{i}$, these projections map chambers to chambers. None of the projections $\pi_{1}, \ldots, \pi_{n}$ fix the fundamental chamber, and (when $W$ is finite) all of them fix the negative chamber. The correspondence between chambers and Weyl group elements induces an action on the group $W$ itself: this is the usual right regular actions of the 0-Hecke monoid, where $\pi_{i}$ adds a left descent at position $i$ if it is not readily there, and $\bar{\pi}_{i}$ does the converse. This definition can be extended to any root $\alpha$ by calling $\alpha^{\vee}$ a 
descent of $w$ if $w\left(\alpha^{\vee}\right)$ is a negative coroot, and defining $\pi_{\alpha}$ accordingly. The action of the $\pi_{i}$ 's can be depicted by a graph on $W$, with an $i$-arrow from $w$ to $w^{\prime}$ if $\pi_{i}(w)=w^{\prime}$. Examples of such graphs are given in Figure 1

Except for the nilCoxeter algebra $\left(q_{1}=q_{2}=0\right)$, the Hecke algebra $\mathrm{H}(W)\left(q_{1}, q_{2}\right)$ can be realized as acting on $\mathbb{C} W$ by interpolation, mapping $T_{i}$ to $\left(q_{1}+q_{2}\right) \pi_{i}-q_{1} s_{i}$. Indeed, by identifying each $w \in \mathbb{C} W$ with $T_{w}$, one recovers the right regular representation of $\mathrm{H}(W)\left(q_{1}, q_{2}\right)$, where

$$
T_{w} T_{i}= \begin{cases}\left(q_{1}+q_{2}\right) T_{w}-q_{1} T_{w s_{i}} & \text { if } i \text { descent of } w \\ q_{2} T_{w s_{i}} & \text { otherwise. }\end{cases}
$$

Through this mapping, $\bar{T}_{i}=\left(q_{1}+q_{2}\right) \bar{\pi}_{i}-q_{2} s_{i}$.

\subsection{Hecke group algebras}

Let $W$ be a finite Coxeter group, and $\mathbb{C} W$ the vector space of dimension $|W|$ it spans. As we have just seen, we may embed simultaneously the Hecke algebra $\mathrm{H}(W)(0)$ and the group algebra $\mathbb{C}[W]$ in End $(\mathbb{C} W)$, via their right regular representations. The Hecke group algebra $\mathrm{HW}$ of $W$ is the smallest subalgebra of $\operatorname{End}(\mathbb{C} W)$ containing them both (see [HT07]). It is therefore generated by $\left(\pi_{i}\right)_{i \in I}$ and $\left(s_{i}\right)_{i \in I}$, and by interpolation it contains all $q_{1}, q_{2}$-Hecke algebras but the nilCoxeter algebra. A basis for $\mathrm{H} W$ is given by $\left\{w \pi_{w^{\prime}} \mid \mathrm{D}_{R}(w) \cap \mathrm{D}_{L}\left(w^{\prime}\right)=\emptyset\right\}$. A more conceptual characterization is as follows: call a vector $v$ in $\mathbb{C} W$-left antisymmetric if $s_{i} v=-v$; then, $\mathrm{HW}$ is the subalgebra of $\operatorname{End}(\mathbb{C} W)$ of those operators which preserve all $i$-left antisymmetries.

\section{Affine Weyl groups, Hecke algebras, and their level 0 actions}

Let now $W$ be an affine Weyl group, with index set $I:=\{0, \ldots, n\}$ and Cartan matrix $M$. We always assume that $W$ is irreducible. We denote respectively by $a_{i}$ and $a_{i}^{\vee}$ the coefficients of the canonical linear combination annihilating the columns and rows of $M$, respectively.

The geometric setting described in Section 3.1] differs slightly from the usual one [Kac90]; it turns out to be simpler yet sufficient for our purpose. Note first that $R:=\left\{w\left(\alpha_{i}\right) \mid w \in W, i \in I\right\}$ is the set of real roots; by abuse, we call them roots, as the imaginary roots do not play a role for our purposes. The geometric representations $\mathfrak{h}_{\mathbb{Z}}^{*}$ and $\mathfrak{h}$ defined in Section 2.1 correspond to the root lattice and the coweight space respectively; however, we use neither the inner product, nor the central extension by $c:=\sum_{i=0}^{n} a_{i}^{\vee} \alpha_{i}^{\vee}$. As a consequence, the coroot lattice $\bigoplus_{i \in I} \mathbb{Z} \alpha_{i}^{\vee}$ does not embed faithfully in $\mathfrak{h}_{\mathbb{Z}}$ (since $c=0$ in $\mathfrak{h}_{\mathbb{Z}}$ ), which has the effect of (almost) identifying affine coroots and classical coroots. We also do not make use of the inner product.

\subsection{Affine Weyl groups and alcove walks}

Let $\delta:=\sum_{i \in I} a_{i} \alpha_{i}$ be the so-called null root. The level of an element $x^{\vee}$ of $\mathfrak{h}$ is given by $\ell\left(x^{\vee}\right)=$ $\left\langle x^{\vee}, \delta\right\rangle$; in particular, and by construction, all the coroots are of level 0 . Since $\delta$ is fixed by $W$, the affine hyperplanes $\mathfrak{h}^{\ell}:=\left\{x^{\vee} \mid\left\langle x^{\vee}, \delta\right\rangle=\ell\right\}$ are stabilized by $W$.

At level 0 , the action cl of the affine Weyl group $W$ on $\mathfrak{h}$ reduces to that of a classical finite Weyl group $\stackrel{\circ}{W}:=\operatorname{cl}(W)$; assuming an appropriate labeling of the Dynkin diagram $\stackrel{\circ}{W}=\left\langle s_{1}, \ldots, s_{n}\right\rangle$. This induces a right action of $W$ on $\stackrel{\circ}{W}$ : for $w$ in $\stackrel{\circ}{W}$ and $s_{i} \in W, w \cdot s_{i}:=w \operatorname{cl}\left(s_{i}\right)$, where cl : $W \rightarrow \stackrel{\circ}{W}$ denotes 
the canonical quotient map. We denote respectively by $\stackrel{\circ}{R}$ and $\stackrel{\circ}{R}^{\vee}$ the sets of classical roots and classical coroots.

The reflections in $W$ are given by

$$
\left\{s_{\alpha, m}:=s_{\alpha-m \delta} \mid \alpha \in \stackrel{\circ}{R}^{+} \text {and } m \in c_{\alpha} \mathbb{Z}\right\} .
$$

Here $s_{\alpha, m}$ is the reflection across the hyperplane $H_{\alpha, m}:=H_{\alpha-m \delta}$ along the classical coroot $\alpha^{\vee}$, and $c_{\alpha} \in \mathbb{Q}\left(c_{\alpha}=1\right.$ always in the untwisted case; for the twisted case see Kac [Kac90, Proposition 6.5]). Accordingly, in $\mathfrak{h}_{\mathbb{Z}}$, each coroot $\alpha^{\vee}$ can be written in a unique way as $\alpha^{\vee}=t \operatorname{cl}\left(\alpha^{\vee}\right)$, where $\operatorname{cl}\left(\alpha^{\vee}\right)$ is a classical coroot, and $t \in \mathbb{Q}^{+}$. By construction, $c l$ is compatible with the action: $\operatorname{cl}\left(w\left(\alpha^{\vee}\right)\right)=$ $\operatorname{cl}(w)\left(\operatorname{cl}\left(\alpha^{\vee}\right)\right)$. Furthermore, $\operatorname{cl}\left(\alpha_{i}^{\vee}\right)=\alpha_{i}^{\vee}$ for $i=1, \ldots, n$ and, in the untwisted case $t$ is always 1 and $\operatorname{cl}\left(\alpha_{0}^{\vee}\right)=-\theta^{\vee}$ where $\theta^{\vee}$ is the highest classical coroot.

At level $\ell$, each classical positive root $\alpha$ gives rise to a family $\left(H_{\alpha, m}^{\ell}\right)_{m \in c_{\alpha} \mathbb{Z}}$ of parallel reflection hyperplanes (which all collapse to $H_{\alpha}^{0}$ at level 0):

$$
H_{\alpha, m}^{\ell}:=H_{\alpha-m \delta} \cap \mathfrak{h}^{\ell}=\left\{x^{\vee} \in \mathfrak{h}^{\ell} \mid\left\langle x^{\vee}, \alpha\right\rangle=\ell m\right\} .
$$

The Tits cone is $\left\{x^{\vee} \mid\left\langle x^{\vee}, \delta\right\rangle>0\right\}$, and slicing it at level $\ell>0$ gives rise to the alcove picture. The fundamental alcove $A:=C \cap \mathfrak{h}^{\ell}$ is a simplex, and the alcoves $w(A)$ in its orbit form a tessellation of $\mathfrak{h}^{\ell}$. We call classical fundamental chamber the open simplicial cone $\left\{x^{\vee} \in \mathfrak{h}^{\ell} \mid\left\langle x^{\vee}, \alpha_{i}\right\rangle>0, \forall i=1, \ldots, n\right\}$, and denote by $0^{\ell}$ the intersection point of its walls $\left(H_{\alpha_{i}}^{\ell}\right)_{i=1, \ldots, n}$. Galleries $C, s_{i_{1}}(C), \ldots, s_{i_{1}} \cdots s_{i_{r}}(C)$ induce alcove walks $A, s_{i_{1}}(A), \ldots, s_{i_{1}} \cdots s_{i_{r}}(A)$.

Consider an $i$-crossing for $i \in\{0, \ldots, n\}$ from an alcove $w(A)$ to the adjacent alcove $w s_{i}(A)$, and let $H_{\alpha, m}$ the crossed affine wall. The crossing is positive if $w s_{i}(A)$ is on the positive side of $H_{\alpha, m}$, and negative otherwise.

Remark 3.1 The crossing is positive if and only if $\operatorname{cl}(w)\left(\operatorname{cl}\left(\alpha_{i}^{\vee}\right)\right)$ is a classical negative coroot, that is $i$ is a descent of $\operatorname{cl}(w)$.

Given an alcove path $i_{1}, \ldots, i_{r}$, define $\epsilon_{1}, \ldots, \epsilon_{r}$ by $\epsilon_{k}=1$ if the $k$-th crossing is positive and -1 otherwise. Denote respectively by $\#+=\left|\left\{i \mid \epsilon_{i}=1\right\}\right|$ and $\#-=\left|\left\{i \mid \epsilon_{i}=-1\right\}\right|$ the number of positive and negative crossings. Define the height ht $(w(A))$ of an alcove $w(A)$ with $w=s_{i_{1}} \cdots s_{i_{r}}$ as the (signed) number of hyperplanes $H_{\alpha, m}$ separating $A$ from $w(A)$, where each hyperplane is counted positively if $w(A)$ is on the positive side of $H$ and negatively otherwise. This is given by $\operatorname{ht}(w(A))=\frac{1}{2}(\#+-\#-)$ for any alcove path from $A$ to $w(A)$.

For a simple coroot $\alpha_{i}^{\vee}$, let $c_{i}=c_{\alpha_{i}}$ and define $t_{\alpha_{i}^{\vee}}=s_{\alpha_{i}, c_{i}} s_{\alpha_{i}, 0}$; at level $\ell, t_{\alpha_{i}^{\vee}}$ is the composition of two reflections along parallel hyperplanes, and acts as a translation by $c_{i} \ell \alpha_{i}^{\vee}$. For any $\lambda^{\vee}=\sum_{i=1}^{n} \lambda_{i} \alpha_{i}^{\vee}$ in the classical coroot lattice $\mathfrak{h}_{\mathbb{Z}}^{*}$, set $c\left(\lambda^{\vee}\right)=\sum_{i=1}^{n} c_{i} \lambda_{i} \alpha_{i}^{\vee}$. Then in general, $t_{\lambda \vee}: \mathfrak{h} \rightarrow \mathfrak{h}$ defined by

$$
t_{\lambda \vee}\left(x^{\vee}\right)=x^{\vee}+\ell\left(x^{\vee}\right) c\left(\lambda^{\vee}\right)
$$

belongs to $W$. More specifically, $t_{\lambda \vee}=s_{i_{1}} \cdots s_{i_{r}}$, where $i_{1}, \ldots, i_{r}$ is an alcove walk from $A$ to the translated alcove $t_{\lambda \vee} A$.

Remark 3.2 The height of the alcove $t_{\lambda \vee}(A)$ coincides with the height of the classical coroot $\lambda^{\vee}$, ht $\left(\lambda^{\vee}\right):=$ $\left\langle\lambda^{\vee}, \stackrel{\circ}{\rho}\right\rangle$, where $\stackrel{\circ}{\rho}:=\frac{1}{2} \sum_{\alpha \in \AA^{+}} \alpha$. In particular, a coroot is of height one if and only if it is a simple coroot ( $\rho$ is also the sum of the fundamental weights). 


\subsection{Affine Hecke algebras}

The Hecke algebra $\mathrm{H}(W)\left(q_{1}, q_{2}\right)$ of $W$ is affine. In particular, it is isomorphic to $\mathrm{H}(\stackrel{\circ}{W})\left(q_{1}, q_{2}\right) \otimes \mathbb{C}[Y]$, where $\mathbb{C}[Y]:=\mathbb{C} .\left\{Y^{\lambda^{\vee}} \mid \lambda^{\vee} \in \mathfrak{h}_{\mathbb{Z}}^{\circ}\right\}$ is the group algebra of the coroot lattice. The $Y^{\lambda^{\vee}}$ 's have an expression in terms of the $T_{i}$ 's which generalizes that for translations $t_{\lambda \vee}$ in the affine Weyl group [Mac03, Equation (3.2.10)]:

$$
Y^{\lambda^{\vee}}:=\left(\frac{1}{\sqrt{-q_{1} q_{2}}} T_{i_{1}}\right)^{\epsilon_{1}} \cdots\left(\frac{1}{\sqrt{-q_{1} q_{2}}} T_{i_{r}}\right)^{\epsilon_{r}}=\left(-q_{1} q_{2}\right)^{-\mathrm{ht}\left(\lambda^{\vee}\right)} T_{i_{1}}^{\epsilon_{1}} \cdots T_{i_{r}}^{\epsilon_{r}},
$$

where $i_{1}, \ldots, i_{r}$ is an alcove walk from $A$ to $t_{\lambda}(A)$. The center of $\mathrm{H}(W)\left(q_{1}, q_{2}\right)$ is the subring of invariants $Y^{W}:=\{p \in Y \mid p . w=p\}$. In type $A$, this is the ring of symmetric functions.

As for $W$, the geometric realization at level 0 induces an action cl of the 0 -Hecke monoid $\left\langle\pi_{i} \mid i \in I\right\rangle$ of $W$ on $\stackrel{\mathscr{W}}{\mathrm{O}}$ or equivalently on its classical chambers. Namely, for $w \in \stackrel{\circ}{W}$, and $i \in\{0, \ldots, n\}, w \cdot \pi_{i}:=w$ if $i$ is a descent of $w$ and $w \cdot \pi_{i}:=w s_{i}$ otherwise. This extends for all $q_{1}, q_{2}$ to a representation cl of the affine Hecke algebra $\mathrm{H}(W)\left(q_{1}, q_{2}\right)$ on $\mathbb{C} \stackrel{\circ}{W}_{\text {. }}$

Interestingly enough, and this is the central topic of this paper, the algebra $\operatorname{cl}\left(\mathrm{H}(W)\left(q_{1}, q_{2}\right)\right)$ turns out not to be the classical Hecke algebra $\mathrm{H}(\stackrel{\circ}{W})\left(q_{1}, q_{2}\right)$, except at $q=1$ and certain roots of unity.

\section{Transitivity of the level 0 action of affine 0 -Hecke algebras}

In this section we state and prove the core combinatorial Theorem 4.2 of this paper about transitivity of the level 0 action of affine 0 -Hecke algebras and mention some applications to crystal graphs.

We start with type $A_{n}$ to illustrate the results. Here, each $\pi_{i}$ can be interpreted as a partial (anti)sort operator: it acts on a permutation (or word) $w:=\left(w_{1}, \ldots, w_{n+1}\right)$ by exchanging $w_{i}$ and $w_{i+1}$ if $w_{i}<$ $w_{i+1}$. By bubble sort, any permutation can be mapped via $\pi_{1}, \ldots, \pi_{n}$ to the maximal permutation $w_{0}$, but not conversely. More precisely the (oriented) graph of the action is the usual right permutohedron, which is acyclic with 1 as minimal element and $w_{0}$ as maximal element.

Consider now $w$ as written along a circle, and let $\pi_{0}$ act as above with $i$ taken modulo $n+1$. As suggested by Figure 1 for $n=2$, adding the 0 edges makes the graph of the action strongly connected.

Proposition $4.1 \pi_{0}, \ldots, \pi_{n}$ act transitively on permutations of $\{1, \ldots, n+1\}$.

We start with any permutation $w$ and identify it with $w\left(\varrho^{\vee}\right)=: x^{\vee}=\left(x_{1}, \ldots, x_{n+1}\right)$, where $\varrho^{\vee}=$ $(n+1, \ldots, 1)$.Suppose that the letter $z=n+1$ is at position $k$ in $x^{\vee}$. Then $\pi_{0} \pi_{n} \cdots \pi_{k+1} \pi_{k}\left(x^{\vee}\right)$ has letter $z$ in position 1 . The operator $\tilde{\pi}_{0}$, which acts in the same way as $\pi_{0}$, but only on the last $n$ positions, is defined as follows:

$$
\begin{aligned}
& z x_{1} x_{2} \ldots x_{n-1} x_{n} \\
& x_{1} x_{2} \ldots x_{n-1} z x_{n} \longleftrightarrow \pi_{n-1} \cdots \pi_{1} \\
& x_{1}^{\prime} x_{2} \ldots x_{n-1} z x_{n}^{\prime} \longleftrightarrow \pi_{0} \\
& z x_{2} \ldots x_{n-1} x_{n}^{\prime} x_{1}^{\prime} \longleftrightarrow \pi_{0} \pi_{n} \\
& z x_{1}^{\prime} x_{2} \ldots x_{n-1} x_{n}^{\prime}
\end{aligned}
$$

where $x_{1}^{\prime}=x_{n}$ and $x_{n}^{\prime}=x_{1}$ if $x_{n}>x_{1}$ and $x_{1}^{\prime}=x_{1}$ and $x_{n}^{\prime}=x_{n}$ otherwise. In the last step we have used that the operator $\pi_{0} \pi_{n} \cdots \pi_{1}$ rotates the last $n$ letters cyclically one step to the left, leaving the letter $z$ in position 1 unchanged. 

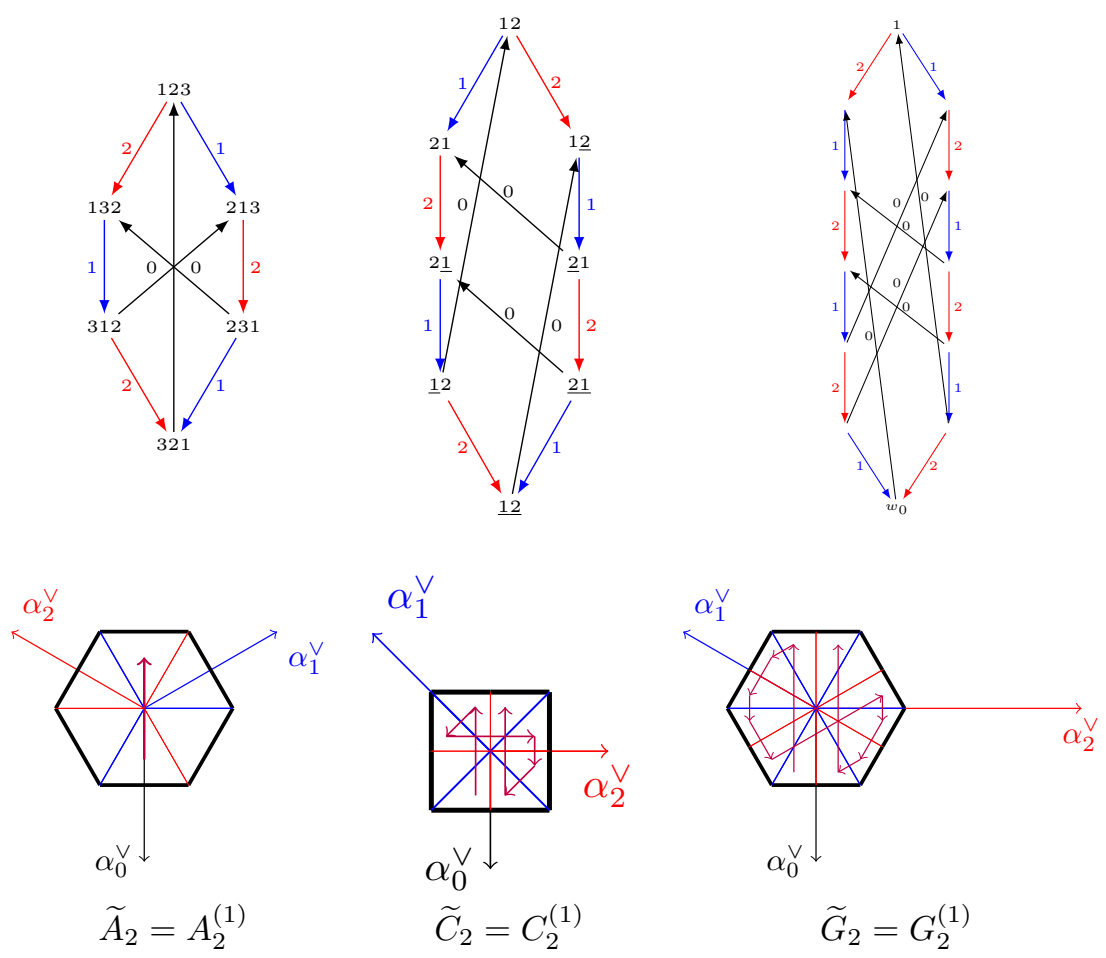

Fig. 1: Top: Graph of the action of $\pi_{0}, \pi_{1}, \ldots, \pi_{n}$ on the classical Weyl group $\stackrel{\circ}{W}$, using (signed) permutation notation. Bottom: The top graph can be realized geometrically by quotienting the alcove picture by the translations, or equivalently by identifying opposite edges of the "hexagons". An $i$-arrow in the graph corresponds to a negative $i$-crossing. The alcove walk goes from the antifundamental chamber $w_{0}(\mathrm{~A})$ back the fundamental chamber $A$.

Let now $\stackrel{\circ}{W}$ be any finite Weyl group, and $\mathrm{H}(\stackrel{\circ}{W})(0)$ its 0 -Hecke algebra. Via $\pi_{1}, \ldots, \pi_{n}$ the identity of $\stackrel{\circ}{W}$ can be mapped to any $w \in \stackrel{\circ}{W}$, but not back (the graph of the action is just the Hasse diagram of the right weak Bruhat order). Embed now $W$ in an affine Weyl group $W$, and consider the extra generator $\pi_{0}$ of its 0-Hecke algebra acting on $W^{\circ}$. As the classical dominant chamber is on the negative side of $H_{\alpha_{0}}, \pi_{0}$ tends to map elements of $W$ back to the identity (see Figure 1 .

Theorem 4.2 Let $W$ be an affine Weyl group, $\stackrel{\circ}{W}$ the associated classical Weyl group, and $\pi_{0}, \pi_{1}, \ldots, \pi_{n}$ the generators of the 0 -Hecke algebra of $W$. Then, the level 0 action of $\pi_{0}, \pi_{1}, \ldots, \pi_{n}$ on $W$ (or equivalently on the classical chambers) is transitive.

We first prove Theorem 4.2 by a type free geometric argument using Lemma 4.3 below. Figure 1 illustrates the proof, actually covering all the rank 2 affine Weyl groups.

Lemma 4.3 (Cf. Remark 3.5 of [ [Ram06]) Let $w(A)$ be an alcove in the classical dominant chamber, and consider a shortest alcove path $i_{1}, \ldots, i_{r}$ from $A$ to $w(A)$. Then, each crossing is positive. In particular, $i_{k}$ is a descent of $\operatorname{cl}\left(s_{i_{1}} \cdots s_{i_{k-1}}\right)$.

We now exhibit a recursive sorting algorithm for type $B_{n}$, using the usual signed permutation represen- 
tation [BB05, EE98], similar to the recursive sorting algorithm for type $A$ at the beginning of this section. This is an explicit algorithm which achieves the results of Theorem 4.2 (but not necessarily in the most efficient way). Sorting algorithms for type $C_{n}$ and $D_{n}$ are analogous. We have also verified by computer that explicit recursive sorting algorithms exist for the exceptional types; the base cases $B_{2}, B_{3}, C_{2}$, and $D_{3}$ can be worked out explicitly. Details are available upon request.

Let $w$ be a permutation of type $B_{n}$ for $n \geq 4$. As before we identify $w$ with $w\left(\varrho^{\vee}\right)=x^{\vee}=$ $\left(x_{1}, \ldots, x_{n}\right)$. We can bring the maximal letter $z=n$ to any position, as $z$ or $-z$ :

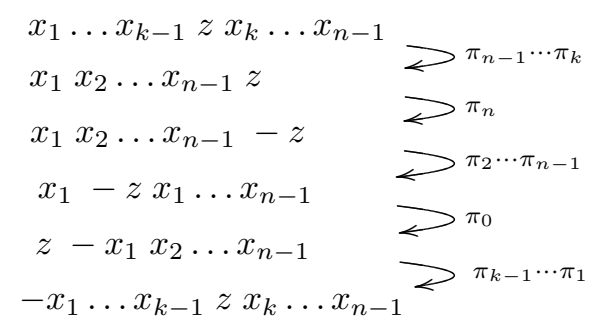

In particular, we can move $z$ to the left of $y=n-1$ (or $-z$ to the right of $-y$ ). The pair $z y$ (or $-y-z$ ) can move around in a circle to any position by similar arguments as above without disturbing any of the other letters, noting that if $z y$ are in the last two positions of $x^{\vee}$, then $\pi_{n} \pi_{n-1} \pi_{n}\left(x^{\vee}\right)$ contains $-y-z$ in the last two positions, and if $-y-z$ is in the first two positions of $x^{\vee}$, then $\pi_{0}\left(x^{\vee}\right)$ contains $z y$ in the first two positions.

Next suppose that $z y$ occupy the first two positions of $x^{\vee}$. We construct $\tilde{\pi}_{0}$ on such $x^{\vee}$, which acts the same way as $\pi_{0}$, but on the last $n-2$ letters:

$$
\begin{aligned}
& z y x_{1} x_{2} \cdots x_{n-2} \\
& x_{1} x_{2} z y \cdots x_{n-2} \\
& x_{1}^{\prime} x_{2}^{\prime} z y \cdots x_{n-2}
\end{aligned} \longleftrightarrow \pi_{2} \pi_{1} \pi_{3} \pi_{2}
$$

followed by the above circling to move $z y$ back to position 1 and 2 .

\section{Alternative generators for Hecke group algebras}

In this section we show that the Hecke group algebra can be entirely generated by $\pi_{0}, \pi_{1}, \ldots, \pi_{n}$.

Proposition 5.1 Let $\stackrel{\circ}{W}$ be a finite Coxeter group, and $\mathcal{S}$ be a set of roots of ${ }^{W}$ such that the associated

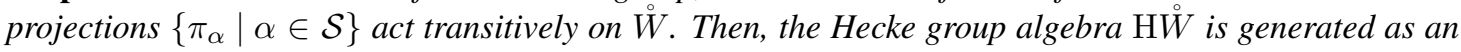
algebra by $\left\{\pi_{\alpha} \mid \alpha \in \mathcal{S}\right\}$.

Corollary 5.2 Let $W$ be an affine Weyl group, $\stackrel{\circ}{W}$ the associated classical group, and $\pi_{0}, \ldots, \pi_{n}$ be the projections associated to the classical roots $\operatorname{cl}\left(\alpha_{0}\right), \ldots, \operatorname{cl}\left(\alpha_{n}\right)$. Then, the Hecke group algebra $\mathrm{H} W^{\circ}$ is generated as an algebra by $\pi_{0}, \ldots, \pi_{n}$.

Alternatively, $\pi_{0}$ may be replaced by any $\Omega \in W$ mapping $\alpha_{0}$ to some simple root, typically one induced by some special Dynkin diagram automorphism. 
Let $w \in \stackrel{\circ}{W}$. An $\mathcal{S}$-reduced word for $w$ is a word $i_{1}, \ldots, i_{r}$ of minimal length such that $i_{j} \in \mathcal{S}$ and $w^{-1} \cdot \pi_{i_{1}} \ldots \pi_{i_{r}}=1$. Since the $\left\{\pi_{\alpha} \mid \alpha \in \mathcal{S}\right\}$ acts transitively on $\stackrel{\circ}{W}$, there always exists such an $\mathcal{S}$-reduced word, and we choose once for all one of them for each $w$. More generally, for a right coset $w \mathscr{W}_{J}$, we choose an $\mathcal{S}$-reduced word $i_{1}, \ldots, i_{r}$ of minimal length such that there exists $\nu \in \stackrel{\circ}{W}_{J} w^{-1}$ and $\mu \in W_{J}$ with $\nu . \pi_{i_{1}} \ldots \pi_{i_{r}}=\mu$.

Example 5.3 In type $C_{2}$, the word $0,1,2,0,1,0$ is $\mathcal{S}$-reduced for $w_{0}=w_{0}{ }^{-1}=(\underline{1}, \underline{2})$, where we write $\underline{1}$ and 2 for -1 and -2 (see Figure 1 ).

In type $A_{3}$ the word 1,0 is $\mathcal{S}$-reduced for $4123 W_{\{1,3\}}$. Here $w=4123, \nu=w^{-1}=2341$, and $\mu=1243$. Looking at $\stackrel{\circ}{W}_{J}$ left-cosets is the Coxeter equivalent to looking at words with repetitions: we may think of left $\dot{W}_{\{1,3\}}$-cosets as identifying the values 1,2 and 3,4 , and represent $\stackrel{\circ}{W}_{\{1,3\}} w^{-1}=2341$ by the word 1331; this word gets sorted by $\pi_{1} \pi_{0}$ to 1133 which represents $\dot{W}_{\{1,3\}}$.

Setting $\nabla_{i}:=\left(\pi_{i}-1\right)$, define the operator $\nabla_{w W_{J}}:=\nabla_{i_{1}} \cdots \nabla_{i_{r}}$ where $i_{1}, \ldots, i_{r}$ is the chosen $\mathcal{S}$-reduced word. The operator may actually depend on the choice of the $\mathcal{S}$-reduced word, but this is irrelevant for our purpose.

Proposition 5.4 The following family forms a basis for $\mathrm{H} \stackrel{\circ}{W}$ :

$$
B:=\left\{\nabla_{w \dot{W}_{\mathrm{D}_{L}\left(w^{\prime}\right)}} \pi_{w^{\prime}} \mid \mathrm{D}_{L}(w) \cap \mathrm{D}_{R}\left(w^{\prime}\right)=\emptyset\right\} .
$$

\section{Hecke group algebras as quotients of affine Hecke algebras}

We now in the position to state the main theorem of this paper. Let $W$ be an affine Weyl group and $\mathrm{H}(W)\left(q_{1}, q_{2}\right)$ its Hecke algebra. Let $\stackrel{\circ}{W}$ be the associated classical group, and $\mathrm{H} \stackrel{\circ}{W}^{2}$ its Hecke group algebra. Then the level 0-representation

$$
\mathrm{cl}: \begin{cases}\mathrm{H}(W)\left(q_{1}, q_{2}\right) & \rightarrow \operatorname{End}(\mathbb{C} \stackrel{\circ}{W}) \\ T_{i} & \mapsto\left(q_{1}+q_{2}\right) \pi_{i}-q_{1} s_{i}\end{cases}
$$

actually defines a morphism from $\mathrm{H}(W)\left(q_{1}, q_{2}\right)$ to $\mathrm{H} \stackrel{\circ}{W}$ (as noted in the proof of Proposition 5.1 all $\pi_{i}$ and $s_{i}$, including $\pi_{0}$ and $s_{0}$, belong to $\mathrm{H} W$ ). When the Dynkin diagram has special automorphisms $\Omega$, this morphism can be extended to the extended affine Hecke algebra by sending the special Dynkin diagram automorphisms to the corresponding element of the finite Weyl group $W$.

Theorem 6.1 Let $W$ be an affine Weyl group. Except when $q_{1}+q_{2}=0$ (and possibly when $q:=-\frac{q_{1}}{q_{2}}$ is a $k$-th root of unity with $\left.k \leq 2 \mathrm{ht}\left(\theta^{\vee}\right)\right)$, the morphism $\mathrm{cl}: \mathrm{H}(W)\left(q_{1}, q_{2}\right) \rightarrow \mathrm{HW}$ is surjective and makes the Hecke group algebra $\mathrm{HW}$ into a quotient of the affine Hecke algebra $\mathrm{H}(W)\left(q_{1}, q_{2}\right)$.

Problem 6.2 Determine for which roots of unity $q$ the morphism $\mathrm{cl}$ is not surjective.

\section{Hecke group algebras and principal series representations of affine Hecke algebras}

Let $t: Y \rightarrow \mathbb{C}^{*}$ be a character of the multiplicative group $Y$ (or equivalently of the additive group $\mathfrak{h}_{\mathbb{Z}}^{*}$ ). It induces a representation $M(t):=t \uparrow_{\mathbb{C}[Y]}^{\mathrm{H}(W)\left(q_{1}, q_{2}\right)}$ called principal series representation of the affine Hecke 
algebra $\mathrm{H}(W)\left(q_{1}, q_{2}\right)$. Since $\mathrm{H}(W)\left(q_{1}, q_{2}\right)=\mathrm{H}(\stackrel{\circ}{W})\left(q_{1}, q_{2}\right) \otimes \mathbb{C}[Y]$, this representation is of dimension $\mid \stackrel{W}{W}$. When $t$ is regular, the representation is calibrated: it admits a basis $\left(E_{w}\right)_{w \in W}$ which diagonalizes the action of $Y$ with a distinct character $w t$ on each $E_{w}$. This basis can be constructed explicitly by means of intertwining operators $\tau_{i}$ which skew commute with the elements of $Y$. We refer to [Ram03, Section 2.5] for details. Note also that the construction of the $\tau_{i}$ operators by deformation of the $T_{i}$ is reminiscent of Yang-Baxter graphs [Las03, § 10.7], in which $t$ corresponds to a choice of spectral parameters.

The main result of this section is that for $q_{1}, q_{2} \neq 0$ and $q$ not a root of unity, there exists a suitable character $t$, such that the level 0 representation of the affine Hecke algebra is isomorphic to the principal series representation $M(t)$ (Theorem 7.1), and to deduce that the morphism cl : $\mathrm{H}(W)\left(q_{1}, q_{2}\right) \mapsto \mathrm{H} W$ is surjective (Theorem 7.4).

Theorem 7.1 Assume $q_{1}, q_{2}$ are such that $q_{1}, q_{2} \neq 0$ and $q:=-\frac{q_{1}}{q_{2}}$ is not a $k$-th root of unity with $k \leq 2 \mathrm{ht}\left(\theta^{\vee}\right)$. Then, the level 0 representation of the affine Hecke algebra $\mathrm{H}(W)\left(q_{1}, q_{2}\right)$ is isomorphic to the principal series representation $M(t)$ for the character $t: Y^{\lambda^{\vee}} \mapsto q^{-\operatorname{ht}\left(\lambda^{\vee}\right)}$.

Note that $t\left(Y^{\alpha_{i}^{\vee}}\right)=q^{-1}$ for any simple coroot. By a result of Kato [Ram03, Theorem 2.12 (c)] one sees right away that $M(t)$ is not irreducible. Note also that this is, up to inversion, the same character as for the action of $\mathbb{C}[Y]$ on the constant Macdonald polynomial 1 [RY08, Equation (3.4)].

Proposition 7.2 Assuming the same conditions as in Theorem 7.1 there exists a basis $\left(E_{w}\right)_{w \in W^{\circ}}$ of $\mathbb{C} \stackrel{W}{W}^{\circ}$ which diagonalizes simultaneously all $Y^{\lambda^{\vee}}$ :

$$
E_{w} \cdot Y^{\lambda^{\vee}}=(w t)\left(Y^{\lambda^{\vee}}\right) E_{w}
$$

where $(w t)\left(Y^{\lambda^{\vee}}\right):=q^{-\operatorname{ht}\left(w\left(\lambda^{\vee}\right)\right)}$.

Corollary 7.3 Each choice of $q_{1}$ and $q_{2}$ as in Theorem 7.1 determines a maximal decomposition of the identity into idempotents in $\mathrm{H} \stackrel{\circ}{W}$.

Theorem 7.4 The morphism cl from the affine Hecke algebra $\mathrm{H}(W)\left(q_{1}, q_{2}\right)$ to the Hecke group algebra $\mathrm{H} \stackrel{\circ}{W}$ is surjective for $q_{1}, q_{2}$ as in Theorem 7.1

\section{Acknowledgements}

We would like to thank Jean-Yves Thibon for suggesting the investigation of the connection between affine Hecke algebras and Hecke group algebras. We are also very grateful to Masaki Kashiwara for sharing his private notes on finite-dimensional representations of quantized affine algebras with us, to Arun Ram for pointing out the link with calibrated representations, and to Francesco Brenti, Christophe Holweg, Mark Shimozono, John Stembridge, and Monica Vazirani for fruitful discussions.

This research was partially supported by NSF grants DMS-0501101, DMS-0652641, and DMS-0652652. It started during a visit of the authors at the University of California, San Diego in 2006, under the kind invitation of Adriano Garsia and Richard and Isabelle Kauffmann. It was completed during the inspiring 2008 MSRI Combinatorial Representation Theory program.

The research was driven by computer exploration using the open-source algebraic combinatorics package MuPAD-Combi nat [HT04]. The pictures have been produced (semi)-automatically, using MuPAD-Combi nat, graphviz, dot2tex, and pgf/tikz. 


\section{References}

[BB05] Anders Björner and Francesco Brenti. Combinatorics of Coxeter groups, volume 231 of Graduate Texts in Mathematics. Springer, New York, 2005.

[BZ77] I. N. Bernstein and A. V. Zelevinsky. Induced representations of reductive $p$-adic groups. I. Ann. Sci. École Norm. Sup. (4), 10(4):441-472, 1977.

[Car86] R. W. Carter. Representation theory of the 0-Hecke algebra. J. Algebra, 104(1):89-103, 1986.

[EE98] Henrik Eriksson and Kimmo Eriksson. Affine Weyl groups as infinite permutations. Electron. J. Combin., 5:Research Paper 18, 32 pp. (electronic), 1998.

[HT04] Florent Hivert and Nicolas M. Thiéry. MuPAD-Combinat, an open-source package for research in algebraic combinatorics. Sém. Lothar. Combin., 51:Art. B51z, 70 pp. (electronic), 2004. http: //mupad-combinat.sf.net/.

[HT06] Florent Hivert and Nicolas M. Thiéry. Representation theories of some towers of algebras related to the symmetric groups and their hecke algebras. In Proceedings of FPSAC'06 San Diego, 2006. arXiv:math/0607391v2 [math.RT].

[HT07] Florent Hivert and Nicolas M. Thiéry. The Hecke group algebra of a Coxeter group and its representation theory. Submitted, arXiv:0711.1561v1 [math.RT], 2007.

[Hum90] James E. Humphreys. Reflection groups and Coxeter groups, volume 29 of Cambridge Studies in Advanced Mathematics. Cambridge University Press, Cambridge, 1990.

[Kac90] Victor G. Kac. Infinite-dimensional Lie algebras. Cambridge University Press, Cambridge, third edition, 1990.

[Kas08] Masaki Kashiwara. Private notes on finite-dimensional representations of quantized affine algebras. Private communication, 2008.

[Las03] Alain Lascoux. Symmetric functions and combinatorial operators on polynomials, volume 99 of CBMS Regional Conference Series in Mathematics. Published for the Conference Board of the Mathematical Sciences, Washington, DC, 2003.

[Lus83] George Lusztig. Singularities, character formulas, and a $q$-analog of weight multiplicities. In Analysis and topology on singular spaces, II, III (Luminy, 1981), volume 101 of Astérisque, pages 208-229. Soc. Math. France, Paris, 1983.

[Mac03] I. G. Macdonald. Affine Hecke algebras and orthogonal polynomials, volume 157 of Cambridge Tracts in Mathematics. Cambridge University Press, Cambridge, 2003.

[Nor79] P. N. Norton. 0-Hecke algebras. J. Austral. Math. Soc. Ser. A, 27(3):337-357, 1979.

[Ram03] Arun Ram. Affine Hecke algebras and generalized standard Young tableaux. J. Algebra, 260(1):367-415, 2003. Special issue celebrating the 80th birthday of Robert Steinberg. 
[Ram06] Arun Ram. Alcove walks, Hecke algebras, spherical functions, crystals and column strict tableaux. Pure Appl. Math. Q., 2(4):963-1013, 2006.

[RY08] Arun Ram and Martha Yip. A combinatorial formula for macdonald polynomials. preprint arXiv:0803.1146 [math.CO], 2008.

[Wik08] Wikipedia, 2008. http://en.wikipedia.org/wiki/Hecke_algebra.

[Zel80] A. V. Zelevinsky. Induced representations of reductive $p$-adic groups. II. On irreducible representations of GL(n). Ann. Sci. École Norm. Sup. (4), 13(2):165-210, 1980. 
